\title{
Numerical methods for computing the Evans function
}

\author{
K. M. $\operatorname{Saad}^{1} \quad$ A. M. El-shrae ${ }^{2}$ \\ (Received 11 January 2011; revised 21 June 2011)
}

\begin{abstract}
This article presents the Lie midpoint method, the fourth order Magnus method and the Gauss-Legendre method for the numerical evaluation of the Evans function, a Wronskian-like determinant which arises in the study of the stability of pulse-type waves. Numerical examples and comparisons illustrate the three methods and their relative merits. The main advance is the ability to compute the Evans function for smaller step sizes. The efficiencies of the various methods are further illustrated by increasing the domain size.
\end{abstract}

\section{Contents}

\section{Numerical methods}

http://anziamj . austms.org.au/ojs/index.php/ANZIAMJ/article/view/3860 gives this article, (C) Austral. Mathematical Soc. 2011. Published July 7, 2011. ISSN 1446-8735. (Print two pages per sheet of paper.) Copies of this article must not be made otherwise available on the internet; instead link directly to this URL for this article. 
2.1 Solution on $[\mathrm{O}, \mathrm{L}] \ldots \ldots$. . . . . . . . . . . . . . E80

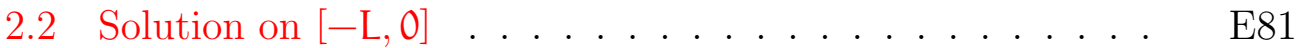

3 The Evans function

E82

4 Applications

E85

4.1 Example 1..................... . . E85

4.2 Example 2..................... E89

5 Conclusions

E94

References

E96

\section{Introduction}

The Evans function, D, is a Wronskian-type analytic function which is widely used to trace the spectrum of ordinary differential operators, $\mathcal{L}_{0}$. Such operators frequently appear due to linearising around exact solutions of partial differential equations. A major aim of such linearisations is to study the stability of such solutions, particularly travelling wave and other solutions which have physical significance. This function was named the Evans function by Alexander, Gardner and Jones [3] who generalized its definition considerably. Since then, the Evans function has been used frequently in stability analyses for numerical computations $[2,4,5,6,10]$ and for analytic treatments $[8,13,19,22,23]$. The review article by Sandstede [20] gives a broad overview of the field.

The zeros of the Evans function give the eigenvalues of the linearised operator, with the order of the zero being the multiplicity of the corresponding eigenvalue. Hence, one obtains information about the spectrum of the operator by finding the zeros of the Evans function, which can be done either analytically or numerically. The focus here is on a numerical approach. In particular, Magnus methods are used to compute the Evans function for spectral problems which 
arise when determining the linear stability of travelling wave solutions to reaction-diffusion and related partial differential equations.

Section 2 presents the Magnus methods. The construction of the Evans function is done in section 3. Finally, section 4 employs Magnus methods to compute the Evans function for spectral problems which arise when determining the linear stability of pulse-type solutions of reaction-diffusion and related partial differential equations. The present methods enable much smaller step sizes to be used than in previous work [16], so that more accurate determinations of the eigenvalues are obtained. Malham and Niesen [16] discussed the numerical evaluation of the Evans function for $h=0.02,0.1$ and 0.2. They did not mention what problems they faced. So we try to examine these methods to the values for smaller step sizes. We also illustrate the efficiencies of the various methods by increasing the domain size. Compared with the work of Malham and Niesen [16], we carry out further computations to examine smaller step sizes than those they considered. In addition, we explore how the size of the truncated domain affects the accuracy of the numerically computed Evans function; Malham and Niesen [16] did not consider this issue. Since the theoretical Evans function is calculated using solutions defined on the unbounded real line, we expect that increasing the size of the domain used for numerical computations will increase the accuracy. This is indeed what we find.

\section{Numerical methods}

Magnus [15] showed that the solution of the linear differential equation $\boldsymbol{u}^{\prime}(x)=$ $A(x) \mathfrak{u}(x)$ can be written in the form $\mathfrak{u}(x)=\exp \{\Omega(x)\} \mathfrak{u}(0)$, where using [., .] to denote the matrix commutator $[A, B]=A B-B A$, the matrix $\Omega(x)$ is the infinite series

$$
\Omega(\xi)=\int_{0}^{\xi} A(x) d x-\frac{1}{2} \int_{0}^{\xi}\left[\int_{0}^{x_{1}} A\left(x_{2}\right) d x_{2}, A\left(x_{1}\right)\right] d x_{1}
$$




$$
\begin{aligned}
& +\frac{1}{12} \int_{0}^{\xi}\left[\int_{0}^{x_{1}} A\left(x_{2}\right) d x_{2},\left[\int_{0}^{x_{1}} A\left(x_{2}\right) d x_{2}, A\left(x_{1}\right)\right]\right] d x_{1} \\
& +\frac{1}{4} \int_{0}^{\xi}\left[\int_{0}^{x_{1}}\left[\int_{0}^{x_{2}} A\left(x_{3}\right) d x_{3}, A\left(x_{2}\right)\right] d x_{2}, A\left(x_{1}\right)\right] d x_{1}+\cdots
\end{aligned}
$$

Moan and Niesen [17] proved that this series converges if $\int_{0}^{x}\|A(\xi)\| d \xi<\pi$. The Magnus series (1) can be used to solve linear differential equations numerically if the infinite series is truncated and the integrals are evaluated numerically. For instance, if we retain only the first term in the series and approximate the integrand $A(x)$ by its value at the midpoint of the range $[0, \xi]$, we obtain $\Omega(\xi) \approx h A(\xi / 2)$, then the resulting one-step method is

$$
\mathfrak{u}_{k+1}=\exp \left\{h A\left(x_{k}+\frac{1}{2} h\right)\right\} \mathfrak{u}_{k},
$$

where $h$ denotes the step size and $\mathfrak{u}_{k}$ is the numerical solution at $x_{k}=x_{0}+k h$. This method is termed the Lie midpoint or exponential midpoint method. A fourth order method is obtained by truncating the Magnus series (1) after the second term. After some manipulation the scheme

$$
\mathfrak{u}_{\mathrm{k}+1}=\exp \left\{\Omega_{\mathrm{k}}\right\} \mathfrak{u}_{\mathrm{k}}
$$

results, where

$$
\Omega_{k}=\frac{1}{2} h\left[A\left(x_{1, k}\right)+A\left(x_{2, k}\right)\right]-\frac{\sqrt{3}}{12} h^{2}\left[A\left(x_{1, k}\right), A\left(x_{2, k}\right)\right] .
$$

The points $x_{1, k}$ and $x_{2, k}$ are the Gauss-Legendre points

$$
x_{1, k}=x_{k}+\left(\frac{1}{2}-\frac{1}{6} \sqrt{3}\right) h, \quad x_{2, k}=x_{k}+\left(\frac{1}{2}+\frac{1}{6} \sqrt{3}\right) h .
$$

Niesen [18] gave details on Magnus methods.

Most reported numerical computations of the Evans function use a RungeKutta method: in particular the classical explicit, fourth order method, and 
the two stage Gauss-Legendre method. As the differential equation we want to solve in the present work is stiff, we use the two stage Gauss-Legendre method. This method is

$$
\mathfrak{u}_{k+1}=\mathfrak{u}_{k}+\frac{1}{2} h\left(S_{1}+S_{2}\right)
$$

where

$$
S_{1}=A\left(x_{1, k}\right)\left\{u_{k}+\frac{1}{4} h S_{1}+\left(\frac{1}{4}-\frac{\sqrt{3}}{6}\right) h S_{2}\right\},
$$

and

$$
S_{2}=A\left(x_{2, k}\right)\left\{u_{k}+\frac{1}{4} h S_{2}+\left(\frac{1}{4}+\frac{\sqrt{3}}{6}\right) h S_{1}\right\} .
$$

Let us now consider the application of this numerical scheme on the intervals $[-\mathrm{L}, 0]$ and $[0, \mathrm{~L}]$, with the step size $\mathrm{h}=\mathrm{L} / \mathrm{N}$, where $\mathrm{N}$ is the number of points used to discretise the interval.

\subsection{Solution on $[0, \mathrm{~L}]$}

To compute the solution $\boldsymbol{u}_{+}$satisfying the right hand boundary condition we shall use and compare the exponential midpoint method, the fourth order Magnus method, and the fourth order Gauss-Legendre method. In detail, the midpoint method is

$$
\mathfrak{u}_{k+1}=\exp \left\{-h A\left(x_{k}-h / 2\right)\right\} \mathfrak{u}_{k},
$$

the fourth order Magnus method is

$$
\mathfrak{u}_{\mathrm{k}+1}=\exp \left\{\Omega_{\mathrm{k}}\right\} \mathfrak{u}_{\mathrm{k}},
$$

where

$$
\Omega_{k}=-\frac{1}{2} h\left\{A\left(x_{1, k}\right)+A\left(x_{2, k}\right)\right\}-\frac{\sqrt{3}}{12} h^{2}\left[A\left(x_{1, k}\right), A\left(x_{2, k}\right)\right],
$$


and the fourth order Gauss-Legendre method is

$$
u_{k+1}=\left\{1+\frac{6 h\left(A\left(x_{1, k}\right)+A\left(x_{2, k}\right)\right)}{12-3 A\left(x_{1, k}\right) h-3 A\left(x_{2, k}\right) h+A\left(x_{1, k}\right) A\left(x_{2, k}\right) h^{2}}\right\} u_{k} .
$$

In these schemes

$$
x_{i, k}=x_{k}-\left(\frac{1}{2} \mp \frac{\sqrt{3}}{6}\right) h, \quad i=1,2, \quad k=1,2, \ldots, N,
$$

and

$$
x_{k}=x_{0}-k h, \quad x_{0}=L, \quad k=1,2, \ldots, N
$$

\subsection{Solution on $[-\mathrm{L}, 0]$}

To compute the solution $\boldsymbol{u}_{-}$satisfying the left hand boundary condition, we again use and compare the exponential midpoint method, the fourth order Magnus method, and the fourth order Gauss-Legendre method. As in the previous subsection, the midpoint method is

$$
\mathfrak{u}_{\mathrm{k}+1}=\exp \left\{\mathrm{hA}\left(\mathrm{x}_{\mathrm{k}}+\mathrm{h} / 2\right)\right\} \mathfrak{u}_{\mathrm{k}},
$$

the fourth order Magnus method is

$$
\mathfrak{u}_{\mathrm{k}+1}=\exp \left\{\Omega_{\mathrm{k}}\right\} \mathfrak{u}_{\mathrm{k}},
$$

where

$$
\Omega_{k}=\frac{1}{2} h\left\{A\left(x_{1, k}\right)+A\left(x_{2, k}\right)\right\}-\frac{\sqrt{3}}{12} h^{2}\left[A\left(x_{1, k}\right), A\left(x_{2, k}\right)\right],
$$

and the Gauss-Legendre method is

$$
u_{k+1}=\left\{1-\frac{6 h\left(A\left(x_{1, k}\right)+A\left(x_{2, k}\right)\right)}{12+3 A\left(x_{1, k}\right) h+3 A\left(x_{2, k}\right) h+A\left(x_{1, k}\right) A\left(x_{2, k}\right) h^{2}}\right\} u_{k} .
$$


Here

$$
x_{i, k}=x_{k}+\left(\frac{1}{2} \mp \frac{\sqrt{3}}{6}\right) h, \quad i=1,2, \quad k=1,2, \ldots, N
$$

and

$$
x_{k}=x_{0}+k h, \quad x_{0}=-L, \quad k=1,2, \ldots, N .
$$

\section{The Evans function}

Let us consider the specific case of a reaction-diffusion equation on an unbounded one-dimensional domain. Such equations have the general form

$$
u_{t}=K u_{x x}+f(u),
$$

where $\mathrm{K}$ is an $\mathrm{n} \times \mathrm{n}$ diagonal matrix with positive entries (the diffusion coefficients), and the function $f: \mathbf{R}^{\mathfrak{n}} \rightarrow \mathbf{R}^{\mathfrak{n}}$ describes the reaction term. The steady state solution $\mathfrak{u}_{0}$ is the solution of

$$
K\left(u_{0}(x)\right)_{x x}+f\left(u_{0}(x)\right)=0 .
$$

We assume the limits $\mathfrak{u}_{0 \pm}=\lim _{x \rightarrow \pm \infty} \mathfrak{u}_{0}(x)$ exist. For a front-type wave we have $\mathfrak{u}_{0_{+}} \neq \mathfrak{u}_{0_{-}}$, whereas pulse-type solutions have $\mathfrak{u}_{0_{+}}=\mathfrak{u}_{0_{-}}$. To study the stability of these solutions, we perturb about the steady state

$$
u(x, t)=u_{0}(x)+\epsilon u(x) e^{\lambda t}+O\left(\epsilon^{2}\right) .
$$

Substituting (23) into (21) gives, to first order in $\epsilon$,

$$
\lambda \mathfrak{u}=K u_{x x}+f_{u}\left(u_{0}(x)\right) u=: \mathcal{L}_{o} u .
$$

The stability of the steady state is then determined by the spectrum of the operator $\mathcal{L}_{0}$. The steady state is unstable if there exists $\lambda \in \mathrm{C}$ such that $\mathfrak{R} \lambda>0$. The spectrum $\sigma$ is defined as the set of all $\lambda \in \mathrm{C}$ such that $\mathcal{L}_{\mathrm{o}}-\lambda \mathrm{I}$ is not boundedly invertible [1,20]; that is, either the inverse operator does 
not exist or it is not bounded. The spectrum is broken down into the essential spectrum

$$
\sigma_{\text {ess }}=\left\{\lambda \in \mathbf{C}: \mathcal{L}_{\mathrm{o}}-\lambda \mathrm{I} \text { is not Fredholm of index zero }\right\}
$$

and the point spectrum $\sigma_{p}=\sigma \backslash \sigma_{\text {ess }}$, which is the set of all isolated eigenvalues of finite multiplicity. The complement of $\sigma$ in $\mathbf{C}$ is the resolvent set of $\mathcal{L}_{\mathrm{o}}$. The spectrum of $\mathcal{L}_{\mathrm{o}}$ has been widely investigated. The essential spectrum has been estimated; for example by Henry [11], and in Smoller's statement of Weyl's Lemma [12]. This estimation can be done using exponential dichotomies for the first order system $(24)[7,21]$. Fredholm properties are also relevant when determining the spectrum of $\mathcal{L}_{\mathrm{o}}[9,21]$. The relation between these two concepts has been studied [14, 21].

The eigenvalue problem (24) can be cast as the first order non-autonomous differential equation

$$
\mathrm{U}_{\mathrm{x}}=\mathrm{A}(\mathrm{x} ; \lambda) \mathrm{U}, \quad \mathrm{u} \in \mathrm{C}^{\mathrm{n}}, \quad-\infty<x<\infty .
$$

Rewrite (25) in the form

$$
\mathrm{U}_{x}=\left(A_{0}(\lambda)+A_{1}(x)\right) \mathrm{U}
$$

with

$$
A_{0}(\lambda)=\left(\begin{array}{cc}
0 & 1 \\
K^{-1} \lambda & 0
\end{array}\right), \quad A_{1}(x)=\left(\begin{array}{cc}
0 & 0 \\
-f_{u}\left(u_{0}(x)\right) & 0
\end{array}\right)
$$

and

$$
\mathrm{u}=(\mathrm{u}, \mathrm{v})^{\top}
$$

Let us first consider the equation

$$
\mathrm{U}_{\mathrm{x}}=\mathrm{A}_{\mathrm{o}}(\lambda) \mathrm{U}, \quad \lambda \in \mathbf{C} .
$$

We assume that $A_{0}(\lambda)$ has eigenvalues with positive real part, say $\mu_{i}^{+}(\lambda)$, for $i=1, \ldots, k$, and eigenvalues with negative real part, say $\mu_{i}^{-}(\lambda)$, for 
$i=k+1, \ldots, n$. The eigenvectors associated with $\mu_{i}^{ \pm}(\lambda)$ are denoted by $\boldsymbol{\eta}_{i}^{ \pm}(\lambda)$. We define the subspaces

$$
\begin{aligned}
& E^{s}=\left\{U_{i}(\lambda) \mid \mathfrak{R}\left(\mu_{i}(\lambda)\right)<0, i=1, \ldots, k\right\}, \\
& E^{\mathfrak{u}}=\left\{U_{i}(\lambda) \mid \mathfrak{R}\left(\mu_{i}(\lambda)\right)>0, i=k+1, \ldots, n\right\}
\end{aligned}
$$

The solutions that belong to $E^{s}$ are bounded as $x \rightarrow \infty$, while the solutions that belong to $E^{\mathfrak{u}}$ are bounded as $x \rightarrow-\infty$. We then label these solutions as $U^{s}(\lambda)$ or $U^{\mathfrak{u}}(\lambda)$ according to whether they are bounded as $x \rightarrow \infty$ or $x \rightarrow-\infty$. These solutions satisfy

$$
\lim _{x \rightarrow-\infty} u_{i}^{s}(x, \lambda) e^{-\mu_{i}^{+}(\lambda) x}=\eta_{i}^{+}(\lambda), \quad i=1, \ldots, k,
$$

and

$$
\lim _{x \rightarrow+\infty} u_{i}^{\mathfrak{u}}(x, \lambda) e^{-\mu_{i}^{-}(\lambda) x}=\eta_{i}^{-}(\lambda), \quad i=k+1, \ldots, n .
$$

To determine the essential spectrum of $\mathcal{L}_{\text {o }}$ we compute $\mathfrak{R}(\mu(\lambda))=0$. The essential spectrum is contained within the parabolic curves of the continuous spectrum. In many cases $\sigma_{\text {ess }}$ is shown to be in the left-half complex plane. In these cases the stability is therefore determined by the position of the point spectrum. To determine this point spectrum we must solve a linear system with non-constant coefficients, which, in general, can only be done numerically.

The Evans function D is defined as the determinant of the Wronskian of the fundamental matrix solution generated by $E^{s}$ and $E^{u}$. In general, this Evans function is nonzero; that is, $E^{s}$ and $E^{\mathfrak{u}}$ together form a basis for the solution space. However, for any specific value of $\lambda$ to be in the point spectrum, $E^{s}$ and $E^{u}$ must be linearly dependent and therefore the Wronskian must be zero. Thus, zeros of the Evans function D correspond to isolated eigenvalues in the point spectrum of the linearised operator [1,3,20]. We further note that any solution that satisfies both boundary conditions at $\pm \infty$ must be lie in $E^{s}(\lambda) \cap E^{\mathfrak{u}}(\lambda)$. 
Now bases for the stable and unstable subspaces are numerically determined in the following way. We calculate the eigenvalues of $A_{0}(\lambda)$ with negative real part and their corresponding eigenvectors. Then, by choosing a sufficiently large $L$ to numerically approximate the infinite domain, we solve the homogeneous equation $U_{x}=\left(A_{0}(\lambda)+A_{1}(x)\right) U$ for $x \in[0, L]$ starting from the right end point $x=L$ with the boundary condition $U(L)=\eta_{i}^{-}(\lambda) e^{\mu_{i}^{-}(\lambda) L}$ for $i=1, \ldots, k$. These solutions at $x=0$ form a basis for $E^{s}$. Similarly, by numerically solving the differential equation for $x \in[-\mathrm{L}, 0]$, we obtain a basis for $E^{u}$.

\section{Applications}

We choose two specific examples to illustrate and compare the use of the exponential midpoint method, the fourth order Magnus method, and the fourth order Gauss-Legendre method for computing the Evans function.

\subsection{Example 1}

Let us consider the one dimensional reaction-diffusion equation

$$
u_{t}=u_{x x}-u+2 u^{3} .
$$

This equation has a steady pulse solution given by $\mathfrak{u}_{0}(x)=\operatorname{sech} x$. Perturbing about this steady solution, we have

$$
u(x, t)=\operatorname{sech} x+\epsilon u(x) e^{\lambda t}+O\left(\epsilon^{2}\right) .
$$

Substituting (33) into (32) gives, to first order in $\epsilon$,

$$
u_{x x}-(\lambda+1) u+6 \operatorname{sech}^{2} x=0 .
$$

The stability of the steady pulse is then determined by the spectrum of the operator $\partial_{x x}-1+6 \operatorname{sech}^{2} x$, consisting of a discrete spectrum and a continuous 
spectrum. As the steady solution $6 \operatorname{sech}^{2} x \rightarrow 0$ as $x \rightarrow \pm \infty$, the location of the continuous spectrum on the spectral plane follows from considering the limits of the operator $\partial_{x x}-1$. Therefore the continuous spectrum lies along the portion of the negative real axis $P=\left\{\lambda \mid \lambda=-\omega^{2}-1, \omega \in \mathbf{R}\right\}$. The transition to instability occurs when discrete eigenvalues move from the left half-plane to the right half-plane.

For the reaction-diffusion equation (32),

$$
A_{0}(\lambda)=\left(\begin{array}{cc}
0 & 1 \\
\lambda+1 & 0
\end{array}\right) \text { and } A_{1}(x)=\left(\begin{array}{cc}
0 & 0 \\
-6 \operatorname{sech}^{2} x & 0
\end{array}\right) .
$$

We now show how the Evans function is used to deduce the above stability result: $\lim _{x \rightarrow \pm \infty} A_{1}(x)=0$ and that this decay is exponential. The eigenvalues of $A_{0}(\lambda)$ are

$$
\mu^{ \pm}(\lambda)= \pm \sqrt{1+\lambda}
$$

and the corresponding eigenvectors are

$$
\eta^{ \pm}(\lambda)=\left(1, \mu^{ \pm}(\lambda)\right)^{\top}
$$

One constructs solutions $\mathbf{U}(x, \lambda)$ of equation (26) which satisfy

$$
\begin{aligned}
& \lim _{x \rightarrow-\infty} U^{s}(x, \lambda) e^{-\mu^{+}(\lambda) x}=\eta^{+}(\lambda), \\
& \lim _{x \rightarrow+\infty} U^{\mathfrak{u}}(x, \lambda) e^{-\mu^{-}(\lambda) x}=\eta^{-}(\lambda) .
\end{aligned}
$$

The Evans function is

$$
\mathrm{D}(\lambda)=\operatorname{det}\left(\mathrm{u}^{\mathrm{s}}, \mathrm{u}^{\mathrm{u}}\right)(x, \lambda) .
$$

By Abel's formula it is independent of $x$ [3].

We now determine a basis of the stable subspace numerically. We take an eigenvalue of $A_{0}(\lambda)$ with negative real part and its corresponding eigenvector. 
Then, by choosing a sufficiently large computational domain length L, we solve the homogeneous equation

$$
u_{x}^{s}=\left(A_{0}(\lambda)+A_{1}(x)\right) U^{s}
$$

for $x \in[0, L]$ starting from the right end point with

$$
\mathrm{U}^{\mathrm{s}}(\mathrm{L})=\eta_{-} e^{\mu_{-} \mathrm{L}} .
$$

We then obtain linearly independent solutions of the differential equation. Their values at $x=0$ form a basis for $E^{s}$. We obtain a basis for the unstable subspace numerically in the following manner. We take an eigenvalue of $A_{0}(\lambda)$ with positive real part and its corresponding eigenvector. Then, by choosing a sufficiently large domain length L, we solve the homogeneous equation

$$
u_{x}^{u}=\left(A_{o}(\lambda)+A_{1}(x)\right) u^{u}
$$

for $x \in[-\mathrm{L}, 0]$ starting with

$$
\mathrm{U}^{\mathrm{u}}(-\mathrm{L})=\eta_{+} e^{\mu_{+}(-\mathrm{L})} .
$$

We then obtain linearly independent solutions of the differential equation and their values at $x=0$ form a basis of $E^{u}$.

Let us now use the exponential midpoint method, the fourth order Magnus method, and the fourth order Gauss-Legendre method to solve (41-44) numerically. With these numerical solutions the Evans function is

$$
\mathrm{D}(\lambda)=\operatorname{det}\left(\begin{array}{ll}
\mathrm{u}^{\mathrm{s}} \mathrm{u}^{\mathfrak{u}} &
\end{array} .\right.
$$

The Evans function for the reaction-diffusion equation (32) for step size $h=0.001$ and $L=10$ is shown in Figure 1. The Evans function has two discrete eigenvalues at $\lambda=0$, due to the translation invariance, and $\lambda=3$. Thus we conclude that the pulse wave is unstable. 

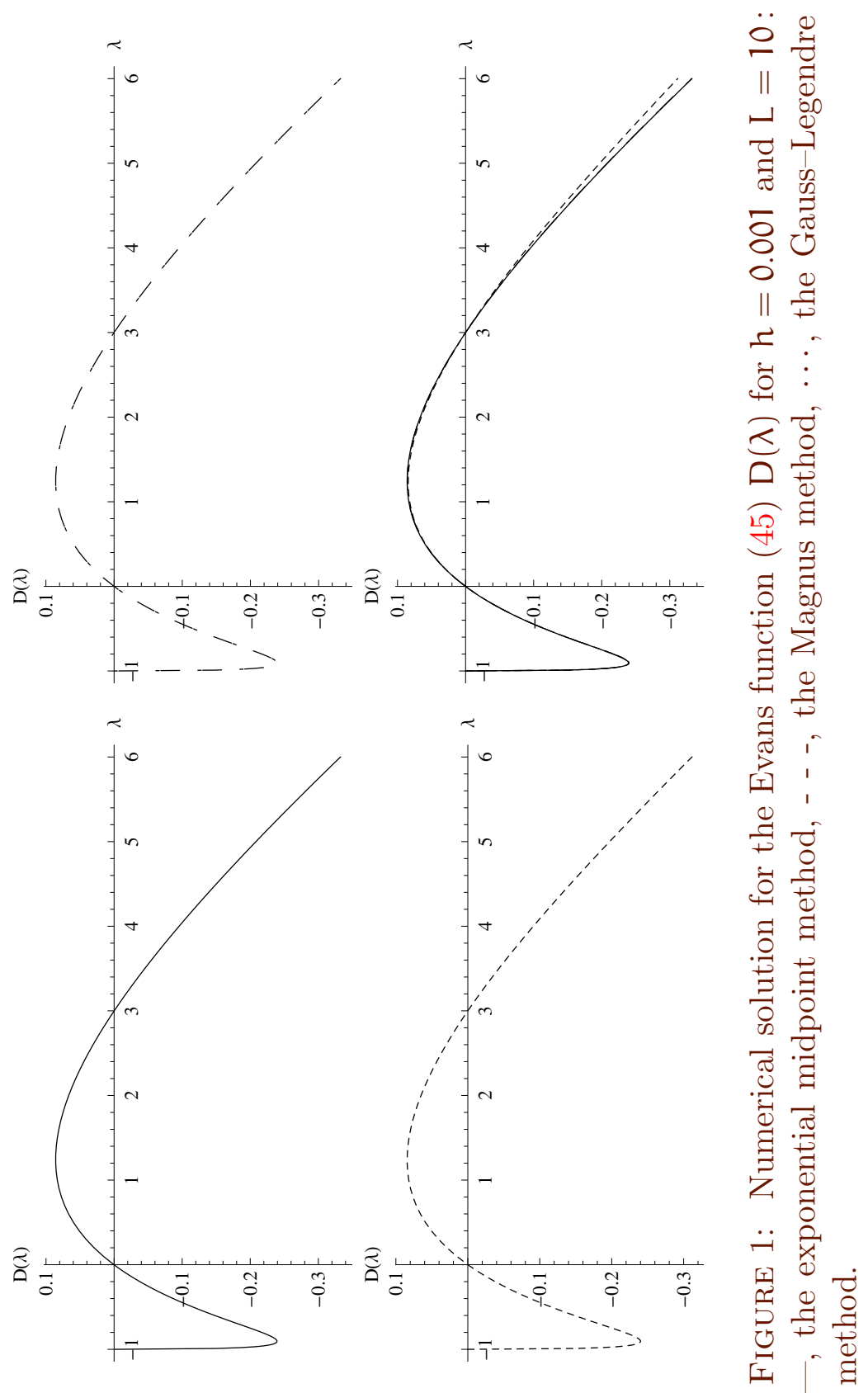
We compute the eigenvalues of (32) analytically. By using the transformation $\xi=\tanh x$ the reaction-diffusion equation (32) becomes the associated Legendre equation

$$
\left(1-\xi^{2}\right) u_{\xi \xi}-2 \xi u_{\xi}+\left(6-\frac{\lambda+1}{1-\xi^{2}}\right) u=0, \quad-1<\xi<1,
$$

of order $m=\sqrt{\lambda+1}$ and degree $v$, where $v(v+1)=6$, so that $v=2$ or -3 . Solutions of this equation have a branch point at $\infty$. The Legendre equation equation (46) has two regular singular points at $\xi= \pm 1$. In terms of the original variable $x$ they correspond to regular singular points of (34) at $x= \pm \infty$. The eigenvalues of (46) are $\sqrt{\lambda+1}=1,2$.

Results are presented in Table 1 to illustrate the error in evaluating the Evans function using the different numerical methods for different values of $h$. Here $E_{\text {exp }}$, $E_{\text {Mag }}$ and $E_{G L}$ denote the absolute errors associated with the exponential midpoint method, the fourth order Magnus method, and the fourth order Gauss-Legendre method, respectively. Malham and Niesen [16] noted that the error in calculating the Evans function using the Magnus method is $\mathrm{O}\left(\lambda^{-1 / 2} h^{4}\right)$, whereas the Gauss-Legendre and the exponential midpoint methods converge more slowly as the error for these method is $\mathrm{O}\left(\lambda^{-1 / 2} h^{2}\right)$ [16]. Malham and Niesen's results confirm this behaviour [16].

In summary, we conclude from Table 1 that

- the Magnus method is more accurate than the other two methods; and

- the results of our numerical computations for the Magnus and the Gauss-Legendre and exponential midpoint methods are consistent with the theoretical results of Malham and Niesen [16].

\section{$4.2 \quad$ Example 2}

Let us now consider the vector (coupled) reaction-diffusion system

$$
u_{\mathrm{t}}=u_{x x}+(1-u) v, \quad v_{t}=v_{x x}-(1-u) v .
$$


TABLE 1: Errors in $D(\lambda)$ for the exponential midpoint method $E_{\text {exp }}$, the Magnus method $E_{\mathrm{Mag}}$ and the Gauss-Legendre method $\mathrm{E}_{\mathrm{GL}}$, respectively, for equation (45) for different values of $h$ and $L=10, \lambda=3$.

\begin{tabular}{lccc}
\hline $\mathrm{h}$ & $\mathrm{E}_{\exp }$ & $\mathrm{E}_{\mathrm{Mag}}$ & $\mathrm{E}_{\mathrm{GL}}$ \\
\hline 0.2 & $7.6 \times 10^{-4}$ & $1.4 \times 10^{-5}$ & $1.5 \times 10^{-5}$ \\
0.05 & $4.7 \times 10^{-5}$ & $5.6 \times 10^{-8}$ & $8.7 \times 10^{-6}$ \\
0.025 & $1.2 \times 10^{-5}$ & $3.5 \times 10^{-9}$ & $4.3 \times 10^{-6}$ \\
0.02 & $7.6 \times 10^{-6}$ & $1.4 \times 10^{-9}$ & $3.2 \times 10^{-6}$ \\
0.01 & $1.9 \times 10^{-6}$ & $8.9 \times 10^{-11}$ & $1.1 \times 10^{-6}$ \\
0.005 & $4.8 \times 10^{-7}$ & $5.6 \times 10^{-12}$ & $3.2 \times 10^{-7}$ \\
0.0025 & $1.2 \times 10^{-7}$ & $3.5 \times 10^{-13}$ & $8.6 \times 10^{-8}$ \\
0.002 & $7.6 \times 10^{-8}$ & $1.4 \times 10^{-13}$ & $5.6 \times 10^{-8}$ \\
0.001 & $1.9 \times 10^{-8}$ & $7.7 \times 10^{-15}$ & $1.4 \times 10^{-8}$ \\
0.0005 & $4.8 \times 10^{-9}$ & $6.8 \times 10^{-16}$ & $3.7 \times 10^{-9}$ \\
0.0002 & $7.6 \times 10^{-10}$ & $1.7 \times 10^{-15}$ & $6.0 \times 10^{-10}$ \\
\hline
\end{tabular}

This coupled system has the pulse solution $u_{0}(x)=\frac{3}{2} \operatorname{sech}^{2} x / 2$ and $v_{0}(x)=$ $-\frac{3}{2} \operatorname{sech}^{2} x / 2$. Equation (26) is now

$$
\mathrm{u}_{\mathrm{x}}=\left(\mathrm{A}_{0}(\lambda)+\mathrm{A}_{1}(\mathrm{x})\right) \mathrm{u}
$$

where

$$
\begin{gathered}
A_{0}(\lambda)=\left(\begin{array}{cccc}
0 & 0 & 1 & 0 \\
0 & 0 & 0 & 1 \\
\lambda & -1 & 0 & 0 \\
0 & 1+\lambda & 0 & 0
\end{array}\right), \\
A_{1}(x)=\left(\begin{array}{cccc}
0 & 0 & 0 & 0 \\
0 & 0 & 0 & 0 \\
-\frac{3}{2} \operatorname{sech}^{2} \frac{x}{2} & \operatorname{sech}^{2} \frac{x}{2} & 0 & 0 \\
\frac{3}{2} \operatorname{sech}^{2} \frac{x}{2} & -\frac{3}{2} \operatorname{sech}^{2} \frac{x}{2} & 0 & 0
\end{array}\right)
\end{gathered}
$$


and

$$
u=\left(\begin{array}{c}
u \\
v \\
u_{x} \\
v_{x}
\end{array}\right)
$$

The eigenvalues of $A_{0}(\lambda)$ are

$$
\mu_{1}^{ \pm}(\lambda)= \pm \sqrt{\lambda} \text { and } \mu_{2}^{ \pm}(\lambda)= \pm \sqrt{1+\lambda}
$$

with the associated eigenvectors

$$
\boldsymbol{\eta}_{1}^{ \pm}=\left(\begin{array}{llll}
1 / \mu_{1}^{ \pm} & 0 & 1 & 0
\end{array}\right)^{\top} \quad \text { and } \quad \boldsymbol{\eta}_{2}^{ \pm}=\left(\begin{array}{llll}
1 / \mu_{2}^{ \pm} & 1 / \mu_{2}^{\mp} & -1 & 1
\end{array}\right)^{\top} .
$$

As for Example 4.1, we use Magnus methods to solve the homogeneous equation (48) for $\mathfrak{R} \lambda>0$. By choosing a sufficiently large domain size $L$ we solve equation (48) for $x \in[0, \mathrm{~L}]$ with the boundary conditions

$$
\mathrm{u}_{1,2}^{s}(\mathrm{~L})=e^{\mu_{1,2}^{-}(\mathrm{L})} \eta_{1,2}^{-},
$$

and (48) for $x \in[-\mathrm{L}, 0]$ with the boundary conditions

$$
\mathrm{U}_{1,2}^{\mathrm{u}}(-\mathrm{L})=\mathrm{e}^{\mu_{1,2}^{+}(-\mathrm{L})} \eta_{1,2}^{+} \text {. }
$$

The solution of these equations at $x=0$ then forms a basis for $E^{s}(\lambda)$ and $E^{u}(\lambda)$. Therefore the Evans function is

$$
\mathrm{D}(\lambda)=\operatorname{det}\left(\begin{array}{llll}
u_{1}^{s} & \mathrm{u}_{2}^{s} & \mathrm{u}_{1}^{u} & \mathrm{u}_{2}^{u}
\end{array}\right) .
$$

An example of an Evans function calculated using this numerical method is shown in Figure 2 for step size $h=0.001$ and $L=10$. This Evans function has one discrete eigenvalue at $\lambda=5 / 4$, so that the steady pulse wave is unstable.

Now if we let $v=-\mathfrak{u}$ in the reaction-diffusion system (47), we obtain

$$
u_{t}=u_{x x}-u(1-u)
$$




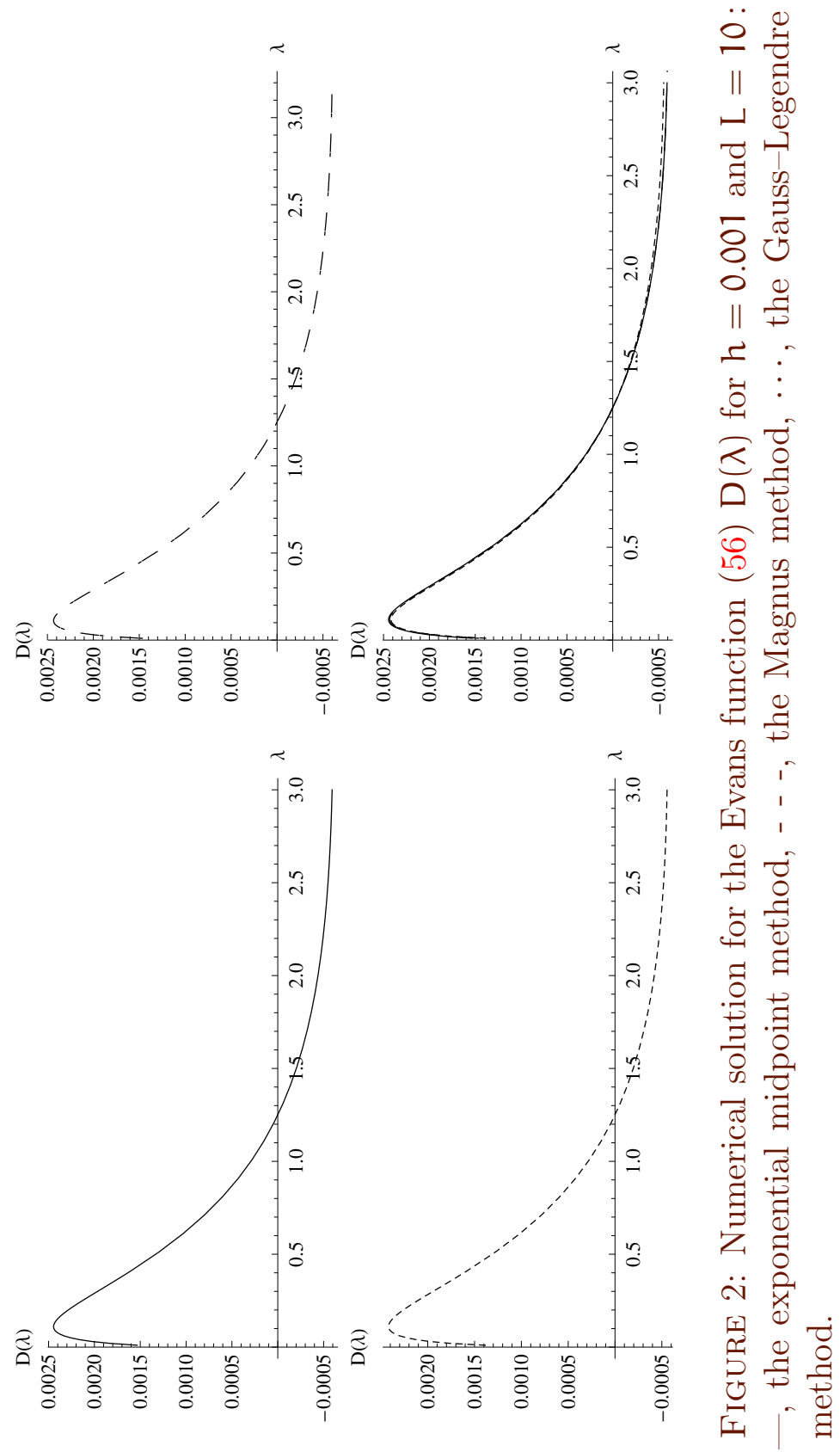


TABLE 2: Errors in $D(\lambda)$ for the exponential midpoint method $E_{\text {exp }}$, the Magnus method $E_{\mathrm{Mag}}$ and the Gauss-Legendre method $E_{\mathrm{GL}}$ for different values of $h$ and $L=10, \lambda=\frac{5}{4}$ for the system (56).

\begin{tabular}{lccc}
\hline $\mathrm{h}$ & $\mathrm{E}_{\exp }$ & $\mathrm{E}_{\mathrm{Mag}}$ & $\mathrm{E}_{\mathrm{GL}}$ \\
\hline 0.2 & $1.4 \times 10^{-6}$ & $8.6 \times 10^{-9}$ & $3.4 \times 10^{-8}$ \\
0.05 & $8.6 \times 10^{-8}$ & $3.5 \times 10^{-11}$ & $3.6 \times 10^{-8}$ \\
0.025 & $2.2 \times 10^{-8}$ & $3.3 \times 10^{-12}$ & $1.7 \times 10^{-8}$ \\
0.02 & $1.2 \times 10^{-8}$ & $2.1 \times 10^{-12}$ & $1.2 \times 10^{-8}$ \\
0.01 & $3.5 \times 10^{-9}$ & $1.3 \times 10^{-12}$ & $4.0 \times 10^{-9}$ \\
0.005 & $8.6 \times 10^{-10}$ & $1.2 \times 10^{-12}$ & $1.1 \times 10^{-9}$ \\
0.0025 & $2.2 \times 10^{-10}$ & $1.2 \times 10^{-12}$ & $3.1 \times 10^{-10}$ \\
0.002 & $1.4 \times 10^{-10}$ & $1.2 \times 10^{-12}$ & $2.0 \times 10^{-10}$ \\
0.001 & $3.6 \times 10^{-11}$ & $1.2 \times 10^{-12}$ & $5.2 \times 10^{-11}$ \\
0.0005 & $9.9 \times 10^{-12}$ & $1.2 \times 10^{-12}$ & $1.4 \times 10^{-12}$ \\
0.0002 & $2.6 \times 10^{-12}$ & $1.2 \times 10^{-12}$ & $3.3 \times 10^{-12}$ \\
\hline
\end{tabular}

As for the reaction-diffusion equation (32), we find the eigenvalue $\lambda$ analytically as $3=2 \sqrt{\lambda+1}$. Table 2 shows the same comparison of the error in the calculation of the Evans function obtained using the three numerical methods as that shown previously in Table 1. Again the Magnus method is the most accurate.

Finally, we investigate the effect of increasing or decreasing the numerical domain length $\mathrm{L}$ on the overall accuracy of the three different schemes. We keep the step size $\mathrm{h}=\mathrm{L} / \mathrm{N}$ fixed by adjusting the number $\mathrm{N}$ of mesh points appropriately for each choice of $\mathrm{L}$ to isolate the the effects of changing the domain length $\mathrm{L}$. The results are shown in Tables 3 and 4: increasing $\mathrm{L}$ decreases the error, as expected. As for the results shown in Tables 1 and 2, the Magnus method is the most accurate. The accuracy of the exponential midpoint and the Gauss-Legendre methods increases to eight decimal places and then does not change as $\mathrm{L}$ is increased further. In contrast, for the Magnus method the error eventually fluctuates around a very small value 
TABLE 3: Errors in $D(\lambda)$ for the exponential midpoint method $E_{\text {exp }}$, the Magnus method $E_{\mathrm{Mag}}$ and the Gauss-Legendre method $E_{\mathrm{GL}}$ for different values of $L$, with $h=0.0005, \lambda=3$ for equation (45).

\begin{tabular}{rrccc}
\hline $\mathrm{N}$ & $\mathrm{L}$ & $\mathrm{E}_{\exp }$ & $\mathrm{E}_{\mathrm{Mag}}$ & $\mathrm{E}_{\mathrm{GL}}$ \\
\hline 2000 & 1 & $2.1 \times 10^{-2}$ & $2.1 \times 10^{-2}$ & $2.1 \times 10^{-2}$ \\
4000 & 2 & $4.9 \times 10^{-5}$ & $4.9 \times 10^{-5}$ & $4.9 \times 10^{-5}$ \\
6000 & 3 & $1.3 \times 10^{-7}$ & $1.2 \times 10^{-7}$ & $1.2 \times 10^{-7}$ \\
8000 & 4 & $5.1 \times 10^{-9}$ & $3.0 \times 10^{-10}$ & $4.0 \times 10^{-9}$ \\
10000 & 5 & $4.8 \times 10^{-9}$ & $7.5 \times 10^{-13}$ & $3.7 \times 10^{-9}$ \\
12000 & 6 & $4.8 \times 10^{-9}$ & $1.6 \times 10^{-15}$ & $3.7 \times 10^{-9}$ \\
14000 & 7 & $4.8 \times 10^{-9}$ & $1.4 \times 10^{-16}$ & $3.7 \times 10^{-9}$ \\
16000 & 8 & $4.8 \times 10^{-9}$ & $8.4 \times 10^{-17}$ & $3.7 \times 10^{-9}$ \\
18000 & 9 & $4.8 \times 10^{-9}$ & $2.5 \times 10^{-16}$ & $3.7 \times 10^{-9}$ \\
20000 & 10 & $4.8 \times 10^{-9}$ & $6.8 \times 10^{-16}$ & $3.7 \times 10^{-9}$ \\
100000 & 50 & $4.8 \times 10^{-9}$ & $6.5 \times 10^{-16}$ & $3.4 \times 10^{-9}$ \\
200000 & 100 & $4.8 \times 10^{-9}$ & $9.7 \times 10^{-16}$ & $3.1 \times 10^{-9}$ \\
400000 & 200 & $4.8 \times 10^{-9}$ & $1.4 \times 10^{-15}$ & $2.5 \times 10^{-9}$ \\
600000 & 300 & $4.8 \times 10^{-9}$ & $1.7 \times 10^{-15}$ & $2.1 \times 10^{-9}$ \\
\hline
\end{tabular}

as L keeps increasing. This suggests that the errors of the midpoint and Gauss-Legendre methods are dominated for lower values of L by the step size, while increasing the domain length $\mathrm{L}$ for the Magnus method gives a much more accurate solution that is limited by $L$ and not by the chosen step size. We conclude that increasing the value of $L$ leads to a more accurate approximation, as expected as the real domain length is infinite.

\section{Conclusions}

We discussed three numerical methods for computing the Evans function. These numerical methods are illustrated by calculating the Evans function for 
TABLE 4: Errors in $D(\lambda)$ for the exponential midpoint method $E_{\text {exp }}$, the Magnus method $E_{\mathrm{Mag}}$ and the Gauss-Legendre method $E_{\mathrm{GL}}$ for different values of $\mathrm{L}$, with $\mathrm{h}=0.0005, \lambda=\frac{5}{4}$ for equation (56).

\begin{tabular}{rrccc}
\hline $\mathrm{N}$ & $\mathrm{L}$ & $\mathrm{E}_{\exp }$ & $\mathrm{E}_{\mathrm{Mag}}$ & $\mathrm{E}_{\mathrm{GL}}$ \\
\hline 2000 & 1 & $1.6 \times 10^{-2}$ & $1.6 \times 10^{-2}$ & $1.6 \times 10^{-2}$ \\
4000 & 2 & $2.3 \times 10^{-4}$ & $2.3 \times 10^{-4}$ & $2.3 \times 10^{-4}$ \\
6000 & 3 & $4.1 \times 10^{-5}$ & $4.1 \times 10^{-5}$ & $4.1 \times 10^{-5}$ \\
8000 & 4 & $4.9 \times 10^{-6}$ & $4.7 \times 10^{-6}$ & $4.7 \times 10^{-6}$ \\
10000 & 5 & $6.1 \times 10^{-7}$ & $4.3 \times 10^{-7}$ & $4.4 \times 10^{-7}$ \\
12000 & 6 & $2.1 \times 10^{-7}$ & $3.6 \times 10^{-8}$ & $3.6 \times 10^{-8}$ \\
14000 & 7 & $1.7 \times 10^{-7}$ & $2.9 \times 10^{-9}$ & $2.8 \times 10^{-9}$ \\
16000 & 8 & $1.7 \times 10^{-7}$ & $2.2 \times 10^{-10}$ & $2.3 \times 10^{-10}$ \\
18000 & 9 & $1.7 \times 10^{-7}$ & $1.7 \times 10^{-11}$ & $2.9 \times 10^{-11}$ \\
20000 & 10 & $1.7 \times 10^{-7}$ & $1.2 \times 10^{-12}$ & $1.4 \times 10^{-11}$ \\
30000 & 15 & $1.7 \times 10^{-7}$ & $3.9 \times 10^{-18}$ & $1.3 \times 10^{-11}$ \\
40000 & 20 & $1.7 \times 10^{-7}$ & $3.9 \times 10^{-19}$ & $1.3 \times 10^{-11}$ \\
100000 & 50 & $1.7 \times 10^{-7}$ & $5.9 \times 10^{-19}$ & $1.2 \times 10^{-11}$ \\
120000 & 60 & $8.6 \times 10^{-12}$ & $2.3 \times 10^{-18}$ & $8.1 \times 10^{-12}$ \\
140000 & 70 & $8.6 \times 10^{-12}$ & $9.5 \times 10^{-19}$ & $3.4 \times 10^{-10}$ \\
160000 & 80 & $7.3 \times 10^{-12}$ & $2.3 \times 10^{-19}$ & $1.7 \times 10^{-8}$ \\
\hline
\end{tabular}

two sample problems. The errors produced in calculating the Evans function using the three different methods were compared and the Magnus method found to be the most accurate. We also considered the effect on the error of increasing the length of the computational domain for equations on the infinite line, which has not been considered in previous studies [16]. Our results again showed that the fourth order Magnus method was the most accurate method. The numerical results presented were done with the aid of Mathematica. 
Acknowledgment We thank Prof. Björn Sandstede for stimulating discussions during the preparation of this article, and Prof. Noel Frederick Smyth for reviewing the language.

\section{References}

[1] N. Valkhoff, Stabilization by competing instability mechanisms, Amsterdam, the Netherlands (2006). http://www.science.uva.nl/ math/Research/Dissertations/Valkhoff2006.text.pdf E82, E84

[2] A. L. Afendikov and T. J. Bridges, Instability of the hockingstewartson pulse and its implications for three-dimensional Poiseuille flow, Proc. $R$. Soc. Lond. A 457 (2001), 257-272. http://epubs.surrey.ac.uk/1407/1/fulltext.pdf E77

[3] J. Alexander, R. Gardner, and C. Jones, A topological invariant arising in the stability analysis of travelling waves, J. Reine Angew. Math. 410 (1990), 167-212. http://www.deepdyve.com/lp/de-gruyter/ a-topological-invariant-arising-in-the-stability-analysis-of$\mathrm{E} 77, \mathrm{E} 84, \mathrm{E} 86$

[4] N. D. Aparicio, S. Malham, and M. Oliver, Numerical evaluation of the Evans function by magnus integration, Proc. $R$. Soc. Lond. BIT 45 (2005), 219-258. http://www.ma.hw.ac.uk/ simonm/magnus.pdf E77

[5] T. J. Bridges, G. Derks, and G. Gottwald, Stability and instability of solitary waves of the fifth-order $\mathrm{kdv}$ equation: A numerical framework, Physica D 172 (2002), 190-216. http://personal.maths.surrey.ac. uk/st/G.Derks/Publications/num_evans.pdf E77

[6] L. Q. Brin, Numerical testing of the stability of viscous shock waves, Math. of Comput. 70 (2000), no. 235, 1071 -1088. E77 
[7] W.A. Coppel, Dichotomies in stability theory, Lect. Notes Math. 629, Springer, 1978. E83

[8] M. Facao and D. F. Parker, Stability of screening solitons in photorefractive media, Phys. Rev E 68 (2003), no. 1, 016610. E77

[9] B. Fiedler and A. Scheel, Spatio-temporal dynamics of reaction-diffusion patterns, in trends in non- linear analysis, Springer-Verlag (2003). http://www.math.umn.edu/ scheel/preprints/tina.pdf E83

[10] V. Gubernov, G. N. Mercer, H. S. Sidhu, and R. O. Weber, Evans function stability of non-adiabatic combustion waves, Proc. $R$. Soc. Lond. A 460 (2004), no. 2048, 24152435.

http://spmlab.phys.msu.su/ vlad/mypapers/ProcRSoc2004.pdf E77

[11] D. Henry, Geometric theory of semilinear parabolic equations, Springer, 1981. E83

[12] J.A.Smoller, Shock waves and reaction diffusion equations, Springer, 1983. E83

[13] T. Kapitula and B. Sandstede, Edge bifurcations for near integrable systems via Evans function techniques, SIAM J. Math. Anal. 33 (2002), no. 5, 1117-1143. http:

//epubs.siam.org/simax/resource/1/sjmaah/v33/i5/p1117_s1 E77

[14] K.J.Palmer, Exponential dichotomies and fredholm operators, Proc. Amer. Math. Soc. 104 (1988), 149-156.

http://www.ams.org/journals/proc/1988-104-01/

S0002-9939-1988-0958058-1/S0002-9939-1988-0958058-1.pdf $\mathrm{E} 83$

[15] W. Magnus, On the exponential solution of differential equations for a linear operator, Comm. Pure and Appl. Math. 7 (1954), 639-673. doi:10.1002/cpa.3160070404 E78 
[16] S. Malham and J. Niesen, Evaluating the Evans function: Order reduction in numerical methods, Math. Comp. 77 (2008), 159-179. doi:10.1090/S0025-5718-07-02016-9 E78, E89, E95

[17] P. C. Moan and J. Niesen, Convergence of the magnus expansion, J. Found. of Comp. Math 8(3) (2008), 291-301.

doi:10.1007/s10208-007-9010-0 E79

[18] J. Niesen, On the global error committed when evaluating the Evans function numerically, Tech. Report HWM 06/43, Heriot-Watt University, 2006.

http://www.amsta.leeds.ac.uk/ jitse/scalarrpt.pdf E79

[19] R. L. Pego and M. I. Weinstein, Eigenvalues, and instabilities of solitary waves, Philos. Trans. R. Soc. Lond A 340 (1992), no. 1, 47-94. E77

[20] B. Sandstede, Stability of travelling waves, Handbook of Dynamical Systems II, (B. Fiedler, ed.), North-Holland, 2002.

http://www.dam.brown.edu/people/sandsted/publications/ survey-stability-of-waves.pdf E77, E82, E84

[21] B. Sandstede and A. Scheel, On the structure of spectra of modulated travelling waves, Math. Nachr. 232 (2001), 39-93. E83

[22] J. Swinton and J. Elgin, Stability of travelling pulse solutions to a laser equation, Phys. Lett. A 145 (1990), no. 8-9, 428-433. http://www. sciencedirect.com/science/article/pii/037596019090307A E77

[23] D. Terman, Stability of planar wave solutions to a combustion model, SIAM J. Math. Anal. 21 (1990), no. 5, 1139-1171. E77

\section{Author addresses}

1. K. M. Saad, Department of Mathematics, Faculty of Arts and Science, Najran University, SAUdi Arabia. On leave from Taiz 
University, YEMEN.

mailto:khaledma_sd@hotmail.com

2. A. M. El-shrae, Department of Mathematics, Faculty of Applied Science, Taiz University, YEMEN.

mailto:elshrae@yahoo.com 